\title{
Extracorporeal membrane oxygenation in COVID-19
}

\author{
Jacek Smereka ${ }^{1}$, Mateusz Puslecki ${ }^{2}$, Kurt Ruetzler ${ }^{3}$, Krzysztof J. Filipiak ${ }^{4}$, \\ Milosz Jaguszewski ${ }^{5}$, Jerzy R. Ladny ${ }^{6}$, Lukasz Szarpak ${ }^{7}$ \\ ${ }^{1}$ Department of Emergency Medical Service, Wroclaw Medical University, Wroclaw, Poland; \\ Polish Society of Disaster Medicine, Warsaw, Poland \\ ${ }^{2}$ Department of Cardiac Surgery and Transplantology, \\ Poznan University of Medical Sciences, Poznan, Poland \\ ${ }^{3}$ Departments of General Anesthesiology and Outcomes Research, Anesthesiology Institute, \\ Cleveland Clinic, Cleveland, OH, United States \\ ${ }^{4} 1^{\text {st }}$ Chair and Department of Cardiology, Medical University of Warsaw, Poland \\ ${ }^{5} 1^{\text {st }}$ Department of Cardiology, Medical University of Gdansk, Poland \\ ${ }^{6}$ Department of Emergency Medicine, Medical University of Bialystok, Poland; \\ Polish Society of Disaster Medicine, Warsaw, Poland \\ ${ }^{7}$ Lazarski University, Warsaw, Poland; Polish Society of Disaster Medicine, Warsaw, Poland
}

This paper was guest edited by Prof. Lukasz K. Czyżewski

An epidemic of severe acute respiratory syndrome coronavirus 2 (SARS-CoV-2) originating in Wuhan, China has, within a short period of time, spread around the world. World Health Organization declared coronavirus disease 2019 (COVID-19) a pandemic. As of April $3^{\text {rd }}, 2020$ there are 1074,017 confirmed cases with 58,004 deaths. Like SARS- $\mathrm{CoV}$ and Middle Eastern respiratory syndrome (MERS)-CoV, SARS-CoV-2 is a coronavirus that can be transmitted to humans. These viruses are all related to high mortality in critically ill patients $[1,2]$. The clinical spectrum of SARS-CoV-2 pneumonia ranges from mild to critically ill cases. The most common symptoms at the onset of COVID-19 illness are fever, cough, and fatigue, while other symptoms include sputum production, headache, hemoptysis, diarrhea, dyspnea, and lymphopenia [3-5].

Mechanical ventilation is the main supportive treatment for critically ill patients. In the case of SARS, the key element of the procedure is additionally low-pressure ventilation strategies with low tidal volume (Vt) ventilation (Vt $4-8 \mathrm{~mL} / \mathrm{kg}$ of predicted body weight) and targeting plateau pressures (Pplat) of $<30 \mathrm{~cm} \mathrm{H}_{2} \mathrm{O}$ and higher positive end-expiratory pressure strategy. This reduces ventilator-induced lung injury [6]. In order to save the lungs and in the era of a limited number of available (unoccupied) ventilators, extracorporeal oxygenation membrane (ECMO) may be helpful in treating patients with COVID-19. ECMO is a resource-intensive technique restricted to specialized centers, and it remains an extremely limited resource. Though there are no clinical trials of ECMO in COVID-19 patients. However a recent report from China suggested that $11.5 \%$ of COVID-19 cases in the intensive care unit received ECMO [7] (however, the results on these patients have not yet been published).

Extracorporeal oxygenation membrane is one of the methods of extracorporeal circulation and ventilation support. For the first time good results of ECMO treatment of acute respiratory failure were published in 1979 in the United States after 15 years of research on its effectiveness. The main objective of ECMO is to oxygenate the blood and eliminate carbon dioxide outside the patient's body. The ECMO system consists of cannulas connecting the extracorporeal circulation with the patient's vascular system, centrifugal pump, oxygenator and drainage system.

Extracorporeal oxygenation membrane therapy can be divided into two methods: venous-venous

Address for correspondence: Lukasz Szarpak, Assoc. Prof. PhD, Lazarski University, ul. Świeradowska 43,

02-662 Warszawa, Poland, tel: +48 500186225, e-mail: lukasz.szarpak@gmail.com

Received: 4.04.2020

Accepted: 10.04.2020 
or venous-arterial. In the case of venous-venous ECMO, blood is collected and donated to the venous system. Most often in the right femoral vein, it is used as a drain of the venous system and the right internal jugular vein is used as the return line to the patient. This variant allows only for respiratory support, which is exactly what is used in patients with COVID-19 who have developed severe respiratory failure. In patients requiring circulatory support, blood is taken from the venous system and returned to the arteries, thus relieving the heart (venous-arterial ECMO).

Combes et al. [8] in EOLIA trial stated that the analysis of the primary end point (mortality at 60 days) in their trial involving patients with very severe acute respiratory distress syndrome showed no significant benefit of early ECMO, as compared with a strategy of conventional mechanical ventilation, which included crossover to ECMO, but when used as a rescue modality ECMO might help improve survival (28\% crossover patients) [8]. In turn, Pham et al. [9] have shown that patients who received ECMO therapy during the influenza A (H1N1) pandemic in 2009 appeared to benefit.

Extracorporeal Life Support Organization (ELSO, Ann Arbor, MI, USA, www.elso.org) is a worldwide non-profit patronage organization of more than 450 centers with units providing almost 130,000 ECMO per year. The main mission of ELSO is to collect registry of ECMO treatments and provide guidelines associated with extracorporeal treatment. Among the indications of ECMO venous-venous therapy lists, among others, extreme respiratory failure not responding to conventional treatment, acute respiratory distress syndrome (including influenza), respiratory disorders in the course of sepsis or asthma. However, contraindications for the use of ECMO therapy should be taken into account, including: contraindications for chronic heparinization, irreversible lesions causing cardiovascular or respiratory failure, or as a relative contraindication - hemorrhagic flaw and/or presence of potential bleeding [10].

Since the beginning of the year, ELSO [11] has been actively uniting specialists from around the world to adapt the inclusion and exclusion criteria to extracorporeal therapies during a pandemic. It must remembered, that in certain healthcare systems, caregivers are challenged to deal with a growing number of patients with a significant reduction in equipment and personnel resources. In such therapies, ECMO therapy may often seem impossible. Therefore, in addition to clinical cri- teria, many centers providing ECMO therapies have to face the introduction of new criteria that take into account limited resources, including age criteria or patient co-morbidities.

Extracorporeal oxygenation membrane remains an option for the treatment of patients with severe respiratory failure in patients in selected age groups with minor co-morbidities. It is very labor-intensive procedure, but may be a life-saving treatment. ECMO will not replace mechanical ventilation or equipment shortages of ventilator availability. ECMO requires supervision by very experienced and well-trained specialist medical personnel, but nevertheless remains a therapeutic option in some well selected patients.

\section{Conflict of interest: None declared}

\section{References}

1. Parry J. Wuhan: Britons to be evacuated as scientists estimate 44000 cases of 2019-nCOV in the city. BMJ. 2020; 368: m351, doi: 10.1136/bmj.m351, indexed in Pubmed: 31996342.

2. Smereka J, Szarpak L, Filipiak KJ. Modern medicine in COVID-19 era. Disaster Emerg Med J. 2020, doi: 10.5603/DEMJ.a2020. 0012 .

3. Huang C, Wang Y, Li X, et al. Clinical features of patients infected with 2019 novel coronavirus in Wuhan, China. Lancet. 2020; 395(10223): 497-506, doi: 10.1016/S0140-6736(20)30183-5, indexed in Pubmed: 31986264.

4. Ruetzler K, Szarpak L, Filipiak KJ, et al. The COVID-19 pandemic - a view of the current state of the problem. Disaster Emerg Med J. 2020, doi: 10.5603/DEMJ.a2020.0015.

5. Smereka J, Szarpak L. COVID 19 a challenge for emergency medicine and every health care professional. Am J Emerg Med. 2020 [Epub ahead of print], doi: 10.1016/j.ajem.2020.03.038, indexed in Pubmed: 32241630.

6. Thompson BT, Chambers RC, Liu KD, et al. Acute respiratory distress syndrome. N Engl J Med. 2017; 377(6): 562-572, doi: 10.1056/NEJMra1608077, indexed in Pubmed: 28792873.

7. Yang X, Yu Y, Xu J, et al. Clinical course and outcomes of critically ill patients with SARS-CoV-2 pneumonia in Wuhan, China: a single-centered, retrospective, observational study. Lancet Respir Med. 2020 [Epub ahead of print], doi: 10.1016/S22132600(20)30079-5, indexed in Pubmed: 32105632.

8. Combes A, Hajage D, Capellier G, et al. Extracorporeal membrane oxygenation for severe acute respiratory distress syndrome. N Engl J Med. 2018; 378(21): 1965-1975, doi: 10.1056/ NEJMoa1800385, indexed in Pubmed: 29791822.

9. Pham T, Combes A, Rozé H, et al. Extracorporeal membrane oxygenation for pandemic influenza $\mathrm{A}(\mathrm{H} 1 \mathrm{~N} 1)$-induced acute respiratory distress syndrome: a cohort study and propensity-matched analysis. Am J Respir Crit Care Med. 2013; 187(3): 276-285, doi: 10.1164/rccm.201205-0815OC, indexed in Pubmed: 23155145.

10. Combes A, Hajage D, Capellier G, et al. Extracorporeal membrane oxygenation for severe acute respiratory distress syndrome. N Engl J Med. 2018; 378(21): 1965-1975, doi: 10.1056/ NEJMoa1800385, indexed in Pubmed: 29791822.

11. https://elso.blog (Access: April 4, 2020). 